\title{
A Dialectical Justification of Inclusion of Ignorance in the Definition of Education
}

\section{Dr. Wycliffe Amukowa}

Senior Lecturer, School of Education

Mount Kenya University, Kenya

Email:kwamukowa@yahoo.com

\section{Caroline Vihenda Ayuya}

Great Lakes University of Kisumu, Kenya Email: ayuyac@yahoo.com

Doi:10.5901/ajis.2013.v2n11p91

Abstract

This paper entertains the notion that without their opposites, things, and by extension, human thought would not exist. Without its opposite aspect, each thing or thought loses the condition for its existence. Without life, there would be no death; without death, there would be no life. Without "above", there would be no "below" without "below", there would be no "above". Without misfortune, there would be no fortune; without fortune, there would be no misfortune. Without facility, there would be no difficulty, without difficulty, there would be no facility. Without landlords, there would be no tenants; without tenants, there would be no landlords. A definition of a thing, entity or a concept necessarily implies its opposite. Reflected alongside this contradiction, education in which its definition bears transmission of knowledge cannot exclude ignorance, which implies the absence of knowledge. Without ignorance, there would be no knowledge, without knowledge, there would be no ignorance. It is the object of this paper to qualify ignorance as a dimension of education, hence its inclusion in the definition of education.

Keywords: Education, Ignorance, Knowledge, Contradiction, Dialectics, Opposites

\section{Introduction}

Zedong (1937) explains that the law of contradiction in things, that is, the law of the unity of opposites, is the fundamental law of nature and of society and therefore also the fundamental law of thought and represents a great revolution in the history of human knowledge. Contradiction is present in all processes of objectively existing things and of subjective thought and permeates all these processes from beginning to end; this is the universality and absoluteness of contradiction. There is nothing that does not contain contradiction; without contradiction nothing would exist. Contradiction is the basis of the simple forms of motion (for instance, mechanical motion) and still more so of the complex forms of motion. Given this observation that contradiction exists in all things and thoughts, this paper seeks an investigation of a contradiction in the concept of education.

The paper kicks off with a conceptualization of education followed by that of ignorance. It then presents the basic tenets of dialectics, being the Law of contradiction and in its later development the paper presents a synthesis of the contradiction in education as a struggle between knowledge and ignorance. Finally, the paper rests with what it considers a balanced definition of education that encompasses ignorance.

\section{Conceptualization of Education}

Scholars provide a variety of the definition of education. Peters (1967) argued that to lay claim to education, first the learner must possess the capability to understand what he is being taught. Second, the process must be done in a manner that is morally acceptable and third, it must be a conscious effort to bring about a positive change in the state of the mind of the recipient which must be directed at achieving a desirable goal. Boyd and King (1977), consider education to be the training and instruction of the young for the business of life. Gwanfogbe (2006) supports this definition of education as given by Boyd and King (1977) and argues that this definition is appropriate because since the beginning of human civilization each human society has been interested in training the future generation to improve on their social, economic, cultural and political life of their society. 
Mohanan (http://www.cdtl.nus.edu.sg/publications/educated/intro.htm) disagrees with the definition of education as training and observes that education is not the same as training, even though training may be one of its ingredients. He argues that for instance, a person who has been taught to repair refrigerators, drive an automobile, or play basketball can be said to have received training, but such training per se does not constitute education. Similarly, even though learning is a necessary ingredient of education, not all forms of learning lead to education. Monkeys, birds, and rats can learn from experience, and they can even be trained, but it cannot be said that they can be educated. Mohanan(ibid) then defines education as the process of actualizing what is unique to the human mental potential. Education enhances the human mental capability because it is a preparation for future life, and a good way of preparing individuals for future life is to enhance their mental capability so that they can cope with the challenges of life more effectively.

According to Balogun (2008), in its etymological derivation, education comes from the Latin word "educere" meaning to "lead out" or "to bring out." Balogun (2008) argues that this definition is sterile as another school of thought has denied that education comes from "educere", to "lead out", but rather from "educare" which means to "form" or "train". This way Balogun (2008) maintains that education refers to the act of developing knowledge, skills or character of a child. It may also be defined as the act of bringing up, rearing, guiding or directing a child.

The 1828 edition of Noah Webster's An American Dictionary of the English Language http://modernedfailedus .blogspot.com/2005/12/times-have-changed.html) presents the definition of Education as the bringing up, as of a child; instruction; formation of manners. However, Omona (1998) argues that education is not only tied to children because adults or persons beyond the connotation of a child continue to be nurtured through education. Indeed, to conceptualize education with focus on a child pays no respect to the present day reality of continuing and adult education.

Indeed in its part of definition of education, the 1983 edition of Webster's New Twentieth Century Dictionary Unabridged Edition, provides that it is the process of 1) training and developing the knowledge, skill, mind, character, etc. especially by formal schooling; teaching; training. 2) Knowledge, ability, etc thus developed, 3) formal schooling.

\section{The Idea of an Educated Person}

According to Schofield (1972), in ancient Greece, an educated person was one who was mentally and physically balanced while in Rome an educated person was one who was a good orator and also excelled in military training. In the Middle Ages England, an educated person was either a lord or priest. Aubery (1999) avers that the educated person (man) is to be discovered by his point of view, by the temper of his mind, by his attitude towards life and his fair of thinking. He can see, he can discriminate, he can combine ideas and see whether they can lead, he has insight and comprehension. He is more apt to contribute light than heat to a discussion and will oftener than not show the power of uniting elements of a difficult subject in a whole view. Peters (1967) held that an educated person is one who posses a considerable body of knowledge together with understanding. He/she has developed capacity to reason to justify his/her beliefs and conduct. The educated person is one who is capable; to certain extend, of doing and knowing things for their own sake.

Mohanan (http://www.cdtl.nus.edu.sg/publications/educated/intro.htm) argues that to be considered educated, a person should have undergone a process of learning that results in enhanced mental capability to function effectively in familiar situations in personal and intellectual life, as well as to adapt to novel situations. This way, an educated person should possess knowledge needed for making informed rational decisions and inferences on familiar and novel situations in personal and intellectual life. In addition an educated person should be able to do certain things. When faced with familiar as well as novel situations, an educated person should be able to perform required tasks, make informed decisions and arrive at informed conclusions. An educated person should have the capability to enhance and modify his/her knowledge and thinking abilities on an ongoing basis so as to cope with novel situations and to cope with them in a more successful manner. An educated person should be capable of independent learning that facilitates coping with and adapting to the changing environment and be capable of using language clearly, precisely and effectively for epistemic purposes.

Michigan State University (www.msu.edu/unit/provost/Educated_Person.htm) in asserting that it is committed to graduating an educated person posts that an educated person is someone who has learned how to acquire, analyze, synthesize, evaluate, understand, and communicate knowledge and information. An educated person has to develop skills that respond to changing professional requirements and new challenges in society and the world at large. He or she must be able to take skills previously gained from serious study of one set of problems and apply them to another. He or she must be able to locate, understand, interpret, evaluate, and use information in an appropriate way and ultimately communicate his or her synthesis and understanding of that information in a clear and accurate manner. Harvard 
University Graduate School of Education (http://www.gse.harvard.edu/news-impact/2012/04/watch-the-askwith-forumlive-defining-the-educated-person/Defining the Educated Person) posts that to be considered educated students should leave school with a deep understanding of themselves and how they fit into the world, and have learned what some call "soft skills" - complex problem-solving, creativity, entrepreneurship, the ability to manage themselves, and the ability to be lifelong learners.

According to Balogun (2008) an educated person shows evidence of well-integrated personality; he or she is morally conscious of his or her actions and shows evidence of responsibility in the social welfare of others. He or she is a person of all season, who is cultured and broadminded; socially sensitive of his or her crucial role in the developmental process, and embraces socialism. Such a person is thus related with society and is an evidential embodiment of societal values with his or her physical body, mind and spirit fully developed to the fullest capacity to ensure the survival of his or her society. In this regard, Balogun (2008) maintained that:

(...) the educated person is one who shows evidence of a well integrated personality, meaning being economically prudent, socially and politically competent, morally acceptable and intellectually and culturally sophisticated. Being economically prudent means being economically efficient in the sense of possessing skills and knowledge that earns a means of survival as well as making a contribution to the common good. Hence a socially and politically competent person is one who has the ability to participate and does participate in decisions as affecting his or her life and others in his or her community. And being ethical, is a function of behavioural dispositions in which makes a person act morally in line with cultural values and norms of society, thus intellectual and cultural sophistication are meant to make the person socially aware of not only the developments in the environment, but also to observe the cultural norms of the society. And thus, a synthesis of all these qualities makes for a true understanding of the idea of educated person (Balogun, 2008:9)

An educated person according to Akinpelu (1969) can be described as one who combines expertise in some specific economic skills with soundness of character and wisdom in judgement. One who is equipped to handle successfully the problems of living in an immediate and an extended family; who is well versed in the folk-lores and genealogies of the ancestors; who has some skills to handle minor health problems and where to obtain advice and help in major ones; who stands well with the ancestral spirits of the family and knows how to observe their worship; who has the ability to discharge social and political duties; who is wise and shrewd in judgement; who expresses self not in too many words but rather in proverbs and analogies leaving hearers to unravel his or her thought; who is self controlled under provocation, dignified in sorrow and restrained in success; and finally and most importantly, who is of excellent character.

\section{Conceptualization of Ignorance}

From Wikipedia, the free encyclopedia, ignorance is a state of being uninformed (lack of knowledge). The word ignorant is an adjective describing a person in the state of being unaware and is often used as an insult to describe individuals who deliberately ignore or disregard important information or facts. Matters which are obvious are sometimes ignored, not taken into consideration. Ignorance can stifle learning, in that a person who falsely believes he or she is knowledgeable will not seek out clarification of his or her beliefs, but rather rely on his or her ignorant position.

Smithson (2008) argues that despite the threat of insoluble problems and paradoxes, it is possible to attain useful knowledge about ignorance. He identifies four characterizations with regard to ignorance:

(...) Ignorance is socially constructed but this realization neither necessitates relativism nor a denial of "real world" influences (...) Ignorance is not always a negative aspect of human affairs. In fact, it is an essential component in social relations, organizations, and culture. People are motivated to create and maintain ignorance, often systematically (...) Ignorance is not invariably a disadvantage for the ignoramus (...) Ignorance is neither marginal nor aberrant in its impact. It is a pervasive and fundamental influence in human cognition, emotion, action, social relations, and culture (Smithson, 2008:209).

Smithson (2008) observed that one difficulty plaguing "ignorance" is that the scattered literature on the topic lacks an agreed-on nomenclature. Here, Smithson (2008) considers several definitions by various scholars.

(...)Böschen and Wehling use the term nichtwissen, whose English equivalent is "nonknowledge."(..) This usage echoes proposals for a "sociology of nonknowledge."(...) A related, if less common, term is nescience (total ignorance). Alternative usages have referred to a social theory of ignorance. (...) Knorr-Cetina introduces the term negative knowledge, that is, knowledge of the limits of knowing, mistakes in attempts to know, things that interfere with knowing, 
and what people do not want to know (...). This concept is quite similar to closed ignorance (...). Outside the social sciences, the most popular general term seems to be uncertainty. For example, this is so in artificial intelligence (...).(Smithson, 2008:209f).

According to Smithson (2008) the main problem in its definition is that anyone referring to ignorance cannot avoid making claims to know something about who is ignorant of what. It probably does not matter greatly what term we choose so long as our definition of it recognizes this point.

\section{Taxonomies of Ignorance}

The intuition that there might be different kinds of ignorance has motivated a number of scholars to propose various distinctions and taxonomies.

(...) One of the most popular distinctions is absence or neglect versus distortion (...). Another popular distinction is reducible versus irreducible ignorance (...)A third, often implicit, distinction is between that which can be known versus that which must not be known (...)active voice (ignoring) from the passive voice (being ignorant). (...) we intentionally close a problem (act of ignoring) or (...) we are unaware of alternative views of the world, or their potential utility (ignorance). (...) ignorance as a lost realm (or selective choice), and ignorance as a deliberate and strategic ploy (active construct) (...) (Smithson, 2008:210).

Some taxonomies of ignorance have emphasized distinctions that operate at a meta-level rather than describing the nature of different kinds of ignorance per se. The most popular distinction is between knowing that we don't know and not knowing that we don't know. In this regard, Smithson (2008) prefers the terms conscious ignorance and metaignorance and argues that several disciplines have produced relatively sophisticated and productive distinctions among special kinds of ignorance and uncertainty. This way, he provides a definition of ignorance as:

"A is ignorant from B's viewpoint if A fails to agree with or show awareness of ideas which $B$ defines as actually or potentially valid." This definition allows $B$ to define what she or he means by ignorance. It also permits self-attributed ignorance, since $A$ and $B$ may be the same person. Most importantly, it incorporates anything $B$ thinks $A$ could or should know (but doesn't) and anything that $B$ thinks $A$ must not know (and doesn't). B's notions about ignorance may be as context-dependent and subjective as required (Smithson, 2008:211).

Whereas it is very difficult to know anything directly about our own or anyone else's ignorance, it is not as hard to find out about people's representations and accounts of ignorance. Ignorance, like knowledge, is largely socially constructed.

Although ignorance may be socially constructed, we should be open-minded about the origins of our primary metaphors for ignorance. After all, some of them appear to be shared with other species and may have been selected in evolutionary processes. The examples for which we have the best evidence of this are the temporal and spatial analogues of uncertainty. Many species (including ours) behave as if events or influences that are nearby or in the near future are more certain than those farther away or further into the future. The underlying metaphor is that certainties are here and now. Uncertainties are later and farther away. Delay is uncertainty. Distance is uncertainty (Smithson, 2008:213).

According to (Smithson, 2008) there are four different kinds of accounts that focus on ignorance.

1. Ignorance as encountered in the external world: Accounts of how ignorance and uncertainty arise in the nonsocial world. These include science as well as epistemological and religious frameworks that make claims about nonknowledge. These accounts make strong claims about meta-knowledge and explain ignorance in exogenous (and usually nonsocial) terms.

2. Ignorance as emergent, constructed, and imposed: Accounts of how ignorance and uncertainty are constructed, imposed, and manipulated by agents. These accounts treat ignorance as at least partly socially constructed. In some cases, ignorance is deliberately or intentionally constructed, whereas in others it emerges as a by-product of some social process.

3. Managing under ignorance: Accounts of how people think and act in uncertain environments. Some of these accounts may invoke or refer to ignorance and uncertainty, but they are not necessarily theories about those topics. 
4. Managing ignorance: Accounts of how people think about ignorance or uncertainty and how they act on it. The distinction between this kind of account and (2) is admittedly fuzzy. Accounts in (2) tend to emphasize the notion that the construction and distribution of knowledge and ignorance are implicated in power relations. Accounts that fall in this fourth category place greater emphasis on individual agency, the micro-level, focusing on how people conceptualize, represent, negotiate, and respond to ignorance.

\section{The Negative Bias Toward Ignorance}

Intellectual culture is predominantly about banishing or reducing ignorance, and negative associations with ignorance are the default. Lakoff, G . Johnson (1980) provide common metaphors for ignorance which negative (Smithson, 2008). For example:

(...) Ignorance is blindness; to know is to see. Or knowledge is power; ignorance is helplessness and impotence, Ignorance is no defense (...) (Lakoff \& Johnson, 1980:10ff).

From either metaphors or theorizations, the underpinning assumption is that ignorance is to be reduced (by gaining knowledge or applying logical systems of rules to quantifying and managing it) or banished altogether. However, according to Smithson (2008), there is a potentially interesting but largely unexplored set of linkages between ignorance (and knowledge), emotional responses, moral assessments, and thereby legitimation. Smithson holds that:

\section{(...) ignorance can be used by the ignoramus as a justification for evading culpability or responsibility. In many cultures, education and other forms of knowledge transmission are moralizing projects; so too are ignorance arrangements such as secrecy, privacy, and the protection of innocence (...) (Smithson, 2008:217).}

\section{A Balanced View of Ignorance}

According to Smithson (2008) Contrary to the view of ignorance and uncertainty as primarily negative, human engagement with ignorance or uncertainty is almost always a mixed-motive enterprise. People sometimes are motivated to discover or create, maintain, and use ignorance (their own as well as others'). Numerous social relations depend on systematic ignorance arrangements. Trust and politeness are obvious examples. The cohesion and smooth operation of many organizations and institutions hinge on ignorance arrangements, and not only (or even typically) for maintaining power differentials.

The very concept of research, for example, presupposes conscious ignorance about the object of research at the outset; otherwise there is nothing to research (Smithson, 2008:218).

Smithson (2008) argues that it is not difficult to find examples of motives for people to remain ignorant about information directly relevant to themselves even when that information is readily available. Majority of parents-to-be not wanting to know the gender of their unborn child, social arrangements such as surprise gift giving, entertainment (for example, spoiling the ending of a novel or movie), and games. These examples highlight the cultural and motivational stock from which people fashion decisions about when to know and when not to. Ignoramuses are not always worse off than knowledgeable folk; in fact there are plenty of contexts in which it can be demonstrated that they are better off. Imagine for a moment that humans were endowed with the ability and a compulsion to indiscriminately absorb all information that came their way and retain all of it for a lifetime. Information acquired decades ago would be as vividly recalled as information acquired seconds ago, so older memories would interfere with more recent and usually more relevant recollections.

\section{Specialization, Privacy, Trust, Politeness, and Legitimation}

Specialization is a social ignorance arrangement. The stereotypical explanation for specialization is that it arises when there is too much for any one person to learn everything. But viewed from an adaptational standpoint, specialization is an example of spreading risk in three respects.

First, the risks of direct learning (versus vicarious learning, which is less risky) are spread across the population by diversifying learning. Second, the risk of being ignorant about crucial matters is spread by diversifying ignorance. Third, the risks associated with bearing knowledge also are diversified. As with any kind of risk spreading, specialization requires various forms of social cooperation to yield these benefits (Smithson, 2008:221). 
Privacy is an example of another kind of social ignorance arrangement. Privacy often has been construed as control over access by others to information, mainly about the self. According to Warren \&Laslett (1977), privacy involves a consensual and essentially cooperative ignorance arrangement, whereas secrecy is unilaterally imposed. Organized specialization and privacy, along with other consensual social ignorance arrangements, are entwined with trust. For instance, effectively functioning expertise requires that nonexperts trust experts to warrant only the knowledge they possess and not to falsify evidence or conclusions within the scope of their expertise. (Smithson, 2008:218).

(...) Trust "entails a state of perceived vulnerability or risk." A primary source of that risk is a requirement that the truster remain partially ignorant about the trustee. Trust is not about concealing information from others, but trust relationships (for example, friendships) do entail a kind of privacy. If a person believes another is monitoring them or insisting that they self-disclose or account for their actions, that person will infer that the other does not trust them (...) (Smithson, 2008:222).

Quoting Yamagishi and his colleagues, Smithson (2008) says that trust and "commitment formation" are alternative ways of reducing the risk of being exploited in social interactions. Commitment formation involves the development of mutual monitoring and powers to sanction and reward each other's behavior. However, the reduction of transaction costs in commitment formation via uncertainty reduction comes at a price, namely the difficulty and costliness in exiting from the relationship and foregoing opportunities for form other relationships. Trust, on the other hand, entails running the risk of being exploited but increases opportunities by rendering the truster more mobile and able to establish cooperative relations more quickly.

Trust, therefore, is both an example of a social relation that requires tolerance of ignorance and also trades undesired uncertainty (the risk of being exploited) against desired uncertainty (freedom to seize opportunities for new relations) (Smithson, 2008:222).

Polite social interaction is another important example of how social relations trade on ignorance. In polite conversation, conversationalists do not expect to deal in the truth, the whole truth, and nothing but the truth. The employment of vagueness and ambiguity in communication serves many of the same purposes in polite conversation as it does in other settings where participants want to promote cooperative goodwill, even if some clarity is sacrificed for it. (Smithson, 2008).

Ignorance as a legitimating influence is used in various guises to justify inaction, maintenance of the status quo, opportunism, evasion of responsibility or culpability, and risk management policies. For example, Western legal traditions distinguish between civil cases in which a guilty verdict may be returned on the "balance of probabilities" and criminal cases wherein guilt must be established "beyond reasonable doubt." In recent times perhaps the premier example of ignorance and uncertainty being used to justify and legitimize high-level policy change in Western countries is the precautionary principle. The precautionary principle essentially stipulates that the burden of proof must not be placed on the environment to show harm in decisions about whether to moderate or halt potentially environmentally damaging activities. Different kinds of ignorance play distinctive roles in both debates and legitimation regarding this principle (Smithson, 2008:224).

\section{Dialectics: The Law of Contradiction in Things}

According to Lenin (1958), dialectics in the proper sense is the study of contradiction in the very essence of objects. Zedong (1937) explains that the law of contradiction in things, that is, the law of the unity of opposites, is the fundamental law of nature and of society and therefore also the fundamental law of thought and represents a great revolution in the history of human knowledge. Zedong avers that:

(...) contradiction is present in all processes of objectively existing things and of subjective thought and permeates all these processes from beginning to end; this is the universality and absoluteness of contradiction (...) The problems are: the two world outlooks, the universality of contradiction, the particularity of contradiction, the principal contradiction and the principal aspect of a contradiction, the identity and struggle of the aspects of a contradiction, and the place of antagonism in contradiction (Zedong,1937:1).

\section{The Two World Outlooks}

According to Zedong (1937), throughout the history of human knowledge, there have been two conceptions concerning the law of development of the universe, the metaphysical conception and the dialectical conception, which form two opposing world outlooks. The two basic conceptions of development are: development as decrease and increase, as 
repetition, and development as a unity of opposites (the division of a unity into mutually exclusive opposites and their reciprocal relation). The metaphysical or vulgar evolutionist world outlook sees things as isolated, static and one-sided. It regards all things in the universe, their forms and their species, as eternally isolated from one another and immutable. Such change as there is can only be an increase or decrease in quantity or a change of place. Moreover, the cause of such an increase or decrease or change of place is not inside things but outside them, that is, the motive force is external.

Metaphysicians hold that all the different kinds of things in the universe and all their characteristics have been the same ever since they first came into being. All subsequent changes have simply been increases or decreases in quantity. They contend that a thing can only keep on repeating itself as the same kind of thing and cannot change into anything different.

They ascribe the causes of social development to factors external to society, such as geography and climate. They search in an over-simplified way outside a thing for the causes of its development, and they deny the theory of materialist dialectics which holds that development arises from the contradictions inside a thing. Consequently they can explain neither the qualitative diversity of things, nor the phenomenon of one quality changing into another (Zedong, 1937:2).

As opposed to the metaphysical world outlook, the world outlook of materialist dialectics holds that in order to understand the development of a thing we should study it internally and in its relations with other things; in other words, the development of things should be seen as their internal and necessary self-movement, while each thing in its movement is interrelated with and interacts on the things around it.

\section{(...)The fundamental cause of the development of a thing is not external but internal; it lies in the contradictoriness within the thing (...) There is internal contradiction in every single thing, hence its motion and development. Contradictoriness within a thing is the fundamental cause of its development, while its interrelations and interactions with other things are secondary causes (Zedong, 1937:2).}

Thus materialist dialectics effectively combats the theory of external causes, or of an external motive force, advanced by metaphysical mechanical materialism and vulgar evolutionism. It is evident that purely external causes can only give rise to mechanical motion, that is, to changes in scale or quantity, but cannot explain why things differ qualitatively in thousands of ways and why one thing changes into another. As a matter of fact, even mechanical motion under external force occurs through the internal contradictoriness of things. Simple growth in plants and animals, their quantitative development, is likewise chiefly the result of their internal contradictions. Similarly, social development is due chiefly not to external but to internal causes. Countries with almost the same geographical and climatic conditions display great diversity and unevenness in their development.

This dialectical world outlook teaches us primarily how to observe and analyse the movement of opposites in different things and, on the basis of such analysis, to indicate the methods for resolving contradictions. It is therefore most important for us to understand the law of contradiction in things in a concrete way (Zedong, 1937:4).

\section{The Universality of Contradiction}

The universality or absoluteness of contradiction has a twofold meaning. One is that contradiction exists in the process of development of all things, and the other is that in the process of development of each thing a movement of opposites exists from beginning to end. The interdependence of the contradictory aspects present in all things and the struggle between these aspects determine the life of all things and push their development forward.

There is nothing that does not contain contradiction; without contradiction nothing would exist. Contradiction is the basis of the simple forms of motion (for instance, mechanical motion) and still more so of the complex forms of motion (Zedong, 1937:4).

In explaining the universality of contradiction, Zedong (1937) observed that if simple mechanical change of place contains a contradiction, this is even more true of the higher forms of motion of matter, and especially of organic life and its development.

Life consists precisely and primarily in this--that a being is at each moment itself and yet something else. Life is therefore also a contradiction which is present in things and processes themselves, and which constantly originates and resolves itself; and as soon as the contradiction ceases, life, too, comes to an end, and death steps in (Zedong, 1937:4).

Engels further explains that also in the sphere of thought we could not escape contradictions, and that for example the contradiction between man's inherently unlimited capacity for knowledge and its actual presence only in men who are 
externally limited and possess limited cognition finds its solution in what is--at least practically, for us-an endless succession of generations, in infinite progress. Lenin (1958) illustrated the universality of contradiction as follows:

\begin{abstract}
(...) In mathematics: + and--. Differential and integral. (...) In mechanics: action and reaction (...) In physics: positive and negative electricity (...) In chemistry: the combination and dissociation of atoms (...) In social science: the class struggle (...) In war, offence and defence, advance and retreat, victory and defeat are all mutually contradictory phenomena. One cannot exist without the other (...)The two aspects are at once in conflict and in interdependence, and this constitutes the totality of a war, pushes its development forward and solves its problems (Quoted in: Zedong, 1937:5).
\end{abstract}

Zedong (1937) discusses that every difference in men's concepts should be regarded as reflecting an objective contradiction. Objective contradictions are reflected in subjective thinking, and this process constitutes the contradictory movement of concepts, pushes forward the development of thought, and ceaselessly solves problems in man's thinking. Opposition and struggle between ideas of different kinds constantly occur within the Party; this is a reflection within the Party of contradictions between classes and between the new and the old in society. If there were no contradictions in the Party and no ideological struggles to resolve them, the Party's life would come to an end. Thus it is already clear that contradiction exists universally and in all processes, whether in the simple or in the complex forms of motion, whether in objective phenomena or ideological phenomena. According to Lenin (1958), contradiction is present in the process of development of all things; it permeates the process of development of each thing from beginning to end which is the universality and absoluteness of contradiction (Zedong, 1937).

\title{
12. The Particularity of Contradiction
}

According to Zedong (1937), the contradiction in each form of motion of matter has its particularity. Man's knowledge of matter is knowledge of its forms of motion, because there is nothing in this world except matter in motion and this motion must assume certain forms. In considering each form of motion of matter, we must observe the points which it has in common with other forms of motion. But what is especially important and necessary, constituting as it does the foundation of our knowledge of a thing, is to observe what is particular to this form of motion of matter, namely, to observe the qualitative difference between this form of motion and other forms.

Only when we have done so can we distinguish between things. Every form of motion contains within itself its own particular contradiction. This particular contradiction constitutes the particular essence which distinguishes one thing from another. It is the internal cause or, as it may be called, the basis for the immense variety of things in the world (Zedong, 1937:7).

There are many forms of motion in nature, mechanical motion, sound, light, heat, electricity, dissociation, combination, and so on. All these forms are interdependent, but in its essence each is different from the others. The particular essence of each form of motion is determined by its own particular contradiction. This holds true not only for nature but also for social and ideological phenomena. Every form of society, every form of ideology, has its own particular contradiction and particular essence (Zedong, 1937).

The sciences are differentiated precisely on the basis of the particular contradictions inherent in their respective objects of study. Thus the contradiction peculiar to a certain field of phenomena constitutes the object of study for a specific branch of science. For example:

\begin{abstract}
(...) positive and negative numbers in mathematics; action and reaction in mechanics; positive and negative electricity in physics; dissociation and combination in chemistry; forces of production and relations of production, classes and class struggle, in social science; offence and defense in military science; idealism and materialism, the metaphysical outlook and the dialectical outlook, in philosophy (...) all these are the objects of study of different branches of science precisely because each branch has its own particular contradiction and particular essence (Zedong, 1937:7).
\end{abstract}

Zedong (1937) emphasizes that unless we understand the universality of contradiction, we have no way of discovering the universal cause or universal basis for the movement or development of things; however, unless we study the particularity of contradiction, we have no way of determining the particular essence of a thing which differentiates it from other things, no way of discovering the particular cause or particular basis for the movement or development of a thing, and no way of distinguishing one thing from another or of demarcating the fields of science.

As regards the sequence in the movement of man's knowledge, there is always a gradual growth from the knowledge of individual and particular things to the knowledge of things in general. Only after man knows the particular essence of many different things can he proceed to generalization and know the common essence of things. When man 
attains the knowledge of this common essence, he uses it as a guide and proceeds to study various concrete things which have not yet been studied, or studied thoroughly, and to discover the particular essence of each; only thus is he able to supplement, enrich and develop his knowledge of their common essence and prevent such knowledge from withering or petrifying.

These are the two processes of cognition: one, from the particular to the general, and the other, from the general to the particular. Thus cognition always moves in cycles and (so long as scientific method is strictly adhered to) each cycle advances human knowledge a step higher and so makes it more and more profound (Zedong, 1937:7).

It is necessary not only to study the particular contradiction and the essence determined thereby of every great system of the forms of motion of matter, but also to study the particular contradiction and the essence of each process in the long course of development of each form of motion of matter. In every form of motion, each process of development which is real (and not imaginary) is qualitatively different. Qualitatively different contradictions can only be resolved by qualitatively different methods:

(...) the contradiction between the proletariat and the bourgeoisie is resolved by the method of socialist revolution; the contradiction between the great masses of the people and the feudal system is resolved by the method of democratic revolution; the contradiction between the colonies and imperialism is resolved by the method of national revolutionary war; the contradiction between the working class and the peasant class in socialist society is resolved by the method of collectivization and mechanization in agriculture; contradiction within the Communist Party is resolved by the method of criticism and self-criticism; the contradiction between society and nature is resolved by the method of developing the productive forces (...)(Zedong, 1937:8).

Processes change, old processes and old contradictions disappear, new processes and new contradictions emerge, and the methods of resolving contradictions differ accordingly. In order to reveal the particularity of the contradictions in any process in the development of a thing, in their totality or interconnections, that is, in order to reveal the essence of the process, it is necessary to reveal the particularity of the two aspects of each of the contradictions in that process; otherwise it will be impossible to discover the essence of the process. To be one-sided means not to look at problems all-sidedly, for example:

(...) to understand only the proletariat but not the bourgeoisie, only the peasants but not the landlords, only the favorable conditions but not the difficult ones, only the past but not the future, only individual parts but not the whole, only the defects but not the achievements, only the plaintiff's case but not the defendant's, only underground revolutionary work but not open revolutionary work, and so on. In a word, it means not to understand the characteristics of both aspects of a contradiction. (...) or it may be called seeing the part but not the whole, seeing the trees but not the forest (...) (Zedong, 1937:9)

To be superficial means to consider neither the characteristics of a contradiction in its totality nor the characteristics of each of its aspects; it means to deny the necessity for probing deeply into a thing and minutely studying the characteristics of its contradiction, but instead merely to look from afar and, after glimpsing the rough outline, immediately to try to resolve the contradiction (to answer a question, settle a dispute, handle work, or direct a military operation).

This way of doing things is bound to lead to trouble. (...) To be one-sided and superficial is at the same time to be subjective. For all objective things are actually interconnected and are governed by inner laws, but instead of undertaking the task of reflecting things as they really are some people only look at things one-sidedly or superficially and who know neither their interconnections nor their inner laws, and so their method is subjectivist (Zedong, 1937:9).

The fundamental contradiction in the process of development of a thing and the essence of the process determined by this fundamental contradiction will not disappear until the process is completed; but in a lengthy process the conditions usually differ at each stage. The reason is that, although the nature of the fundamental contradiction in the process of development of a thing and the essence of the process remain unchanged, the fundamental contradiction becomes more and more intensified as it passes from one stage to another in the lengthy process. In addition, among the numerous major and minor contradictions which are determined or influenced by the fundamental contradiction, some become intensified, some are temporarily or partially resolved or mitigated, and some new ones emerge; hence the process is marked by stages.

If people do not pay attention to the stages in the process of development of a thing, they cannot deal with its contradictions properly (Zedong, 1937:10). 
Because the range of things is vast and there is no limit to their development, what is universal in one context becomes particular in another. Conversely, what is particular in one context becomes universal in another. The relationship between the universality and the particularity of contradiction is the relationship between the general character and the individual character of contradiction. Zedong (1937) explains that:

By the former we mean that contradiction exists in and runs through all processes from beginning to end; motion, things, processes, thinking; all are contradictions. To deny contradiction is to deny everything. This is a universal truth for all times and all countries, which admits of no exception. Hence the general character, the absoluteness of contradiction. But this general character is contained in every individual character; without individual character there can be no general character (...). It is because each contradiction is particular that individual character arises. All individual character exists conditionally and temporarily, and hence is relative (Zedong, 1937:14).

\section{The Principal Contradiction and the Principal Aspect of a Contradiction}

According to Zedong (1937), there are many contradictions in the process of development of a complex thing, and one of them is necessarily the principal contradiction whose existence and development determines or influences the existence and development of the other contradictions. Hence, if in any process there are a number of contradictions, one of them must be the principal contradiction playing the leading and decisive role, while the rest occupy a secondary and subordinate position. The principal aspect is the one playing the leading role in the contradiction. The nature of a thing is determined mainly by the principal aspect of a contradiction, the aspect which has gained the dominant position.

The distinctive character or particularity of these two facets of contradiction represents the unevenness of the forces that are in contradiction. Nothing in this world develops absolutely evenly;

(..) We must oppose the theory of even development or the theory of equilibrium. Moreover, it is these concrete features of a contradiction and the changes in the principal and non-principal aspects of a contradiction in the course of its development that manifest the force of the new superseding the old (Zedong, 1937:18).

\section{The Identity and Struggle of the Aspects of a Contradiction}

Zedong (1937) argues that identity, unity, coincidence, interpenetration, interpermeation, interdependence (or mutual dependence for existence), interconnection or mutual co-operation--all these different terms mean the same thing and refer to the following two points:

(...) first, the existence of each of the two aspects of a contradiction in the process of the development of a thing presupposes the existence of the other aspect, and both aspects coexist in a single entity; second, in given conditions, each of the two contradictory aspects transforms itself into its opposite. This is the meaning of identity (Zedong, 1937:18).

The contradictory aspects in every process exclude each other, struggle with each other and are in opposition to each other. Without exception, they are contained in the process of development of all things and in all human thought. A simple process contains only a single pair of opposites, while a complex process contains more. And in turn, the pairs of opposites are in contradiction to one another. That is how all things in the objective world and all human thought are constituted and how they are set in motion

The fact is that no contradictory aspect can exist in isolation. Without its opposite aspect, each loses the condition for its existence.

Without life, there would be no death; without death, there would be no life. Without "above", there would be no "below" without "below", there would be no "above". Without misfortune, there would be no good fortune; without good fortune, these would be no misfortune. Without facility, there would be no difficulty) without difficulty, there would be no facility. Without landlords, there would be no tenant-peasants; without tenant-peasants, there would be no landlords. Without the bourgeoisie, there would be no proletariat; without the proletariat, there would be no bourgeoisie. Without imperialist oppression of nations, there would be no colonies or semi-colonies; without colonies or semi-colonies, there would be no imperialist oppression of nations. It is so with all opposites; in given conditions, on the one hand they are opposed to each other, and on the other they are interconnected, interpenetrating, interpermeating and interdependent, and this character is described as identity (Zedong, 1937:19).

Zedong (1937) discusses that in given conditions, all contradictory aspects possess the character of non-identity and hence are described as being in contradiction. But they also possess the character of identity and hence are 
interconnected. Because each is the condition for the other's existence they become identical. This is the first meaning of identity. The matter does not end with their dependence on each other for their existence; what is more important is their transformation into each other. That is to say, in given conditions, each of the contradictory aspects within a thing transforms itself into its opposite, changes its position to that of its opposite. This is the second meaning of the identity of contradiction.All contradictory things are interconnected; not only do they coexist in a single entity in given conditions, but in other given conditions, they also transform themselves into each other. This is the full meaning of the identity of opposites.

The fact is that the unity or identity of opposites in objective things is not dead or rigid, but is living, conditional, mobile, temporary and relative; in given conditions, every contradictory aspect transforms itself into its opposite. Why can an egg but not a stone be transformed into a chicken? Why is there identity between war and peace and none between war and a stone? Why can human beings give birth only to human beings and not to anything else? The sole reason is that the identity of opposites exists only in necessary given conditions. Without these necessary given conditions there can be no identity whatsoever (Zedong, 1937:20).

According to Lenin (1958), the unity (coincidence, identity, equal action) of opposites is conditional, temporary, transitory, relative. The struggle of mutually exclusive opposites is absolute, just as development and motion are absolute. Zedong (1937) explains that all processes have a beginning and an end, all processes transform themselves into their opposites. The constancy of all processes is relative, but the mutability manifested in the transformation of one process into another is absolute.

Zedong (1937) discusses that there are two states of motion in all things, that of relative rest and that of conspicuous change. Both are caused by the struggle between the two contradictory elements contained in a thing. When the thing is in the first state of motion, it is undergoing only quantitative and not qualitative change and consequently presents the outward appearance of being at rest. When the thing is in the second state of motion, the quantitative change of the first state has already reached a culminating point and gives rise to the dissolution of the thing as an entity and thereupon a qualitative change ensues, hence the appearance of a conspicuous change. Things are constantly transforming themselves from the first into the second state of motion; the struggle of opposites goes on in both states but the contradiction is resolved through the second state.

(...) That is why we say that the unity of opposites is conditional, temporary and relative, while the struggle of mutually exclusive opposites is absolute (...). (Zedong, 1937:22).

The combination of conditional, relative identity and unconditional, absolute struggle constitutes the movement of opposites in all things. Things that oppose each other also complement each other. That is, things opposed to each other have identity. Oppose each other refers to the mutual exclusion or the struggle of two contradictory aspects. Complement each other means that in given conditions the two contradictory aspects unite and achieve identity. Yet struggle is inherent in identity and without struggle there can be no identity (Zedong, 1937).

The question of the struggle of opposites includes the question of what is antagonism. Antagonism is one form, but not the only form, of the struggle of opposites. Contradiction and struggle are universal and absolute, but the methods of resolving contradictions, that is, the forms of struggle, differ according to the differences in the nature of the contradictions. Some contradictions are characterized by open antagonism, others are not. In accordance with the concrete development of things, some contradictions which were originally non-antagonistic develop into antagonistic ones, while others which were originally antagonistic develop into non-antagonistic ones.

From dialectical account, each contradiction and each of its aspects have their respective characteristics; this is the particularity and relativity of contradiction. In given conditions, opposites possess identity, and consequently can coexist in a single entity and can transform themselves into each other; this again is the particularity and relativity of contradiction. But the struggle of opposites is ceaseless, it goes on both when the opposites are coexisting and when they are transforming themselves into each other, and becomes especially conspicuous when they are transforming themselves into one another; this again is the universality and absoluteness of contradiction.

\section{Education and the Knowledge-Ignorance Dialectical Relationship}

From the conceptualization of education as done in this paper, the observation that education transmits knowledge remains undoubted. Balogun (2008) says that education is an act of developing knowledge. 983 edition of Webster's New Twentieth Century Dictionary Unabridged Edition, provides that it is the process of 1) training and developing the knowledge, skill, mind, character, etc. especially by formal schooling; teaching; training. 2) Knowledge, ability, etc thus 
developed, 3) formal schooling. Definition provided by the other scholars covered in this paper, both have an orientation that implies knowledge as a component of education.

Knowledge as an element of education is revealed also through the idea of an educated person. For instance, Mohanan (ibid) argues that an educated person should have the capability to enhance and modify his/her knowledge and thinking abilities on an ongoing basis so as to cope with novel situations and to cope with them in a more successful manner. Michigan State University (www.msu.edu/unit/provost/Educated_Person.htm) posts that an educated person is someone who has learned how to acquire, analyze, synthesize, evaluate, understand, and communicate knowledge and information. Balogun (2008) holds that an educated person is one who shows evidence of a well integrated personality, meaning being economically prudent, socially and politically competent, morally acceptable and intellectually and culturally sophisticated. Being economically prudent means being economically efficient in the sense of possessing skills and knowledge that earns a means of survival as well as making a contribution to the common good.

From Wikipedia, the free encyclopedia, ignorance is a state of being uninformed (lack of knowledge). ${ }^{[1]}$ The word ignorant is an adjective describing a person in the state of being unaware and is often used as an insult to describe individuals who deliberately ignore or disregard important information or facts. Ignorance can stifle learning, in that a person who falsely believes he or she is knowledgeable will not seek out clarification of his or her beliefs, but rather rely on his or her ignorant position. He or she may also reject valid but contrary information, neither realizing its importance nor understanding it.

Smithson (.n.) provides that ignorance is not always a negative aspect of human affairs. In fact, it is an essential component in social relations, organizations, and culture. People are motivated to create and maintain ignorance, often systematically. Ignorance is not invariably a disadvantage for the ignoramus and it is neither marginal nor aberrant in its impact. It is a pervasive and fundamental influence in human cognition, emotion, action, social relations, and culture.

Ignorance takes on several definitions such as "nonknowledge, negative knowledge, that is, knowledge of the limits of knowing, mistakes in attempts to know, things that interfere with knowing, and what people do not want to know. In its conceptualization of ignorance, this paper shows that outside the social sciences, the most popular general term to define ignorance seems to be uncertainty. Ignorance could also be understood from its taxonomies which this paper shows as follows:

(...) One of the most popular distinctions is absence or neglect versus distortion (...). Another popular distinction is reducible versus irreducible ignorance (...)A third, often implicit, distinction is between that which can be known versus that which must not be known (...)active voice (ignoring) from the passive voice (being ignorant). (...) we intentionally close a problem (act of ignoring) or (...) we are unaware of alternative views of the world, or their potential utility (ignorance). (...) ignorance as a lost realm (or selective choice), and ignorance as a deliberate and strategic ploy (active construct) (...).(Smithson, ibid)

Common metaphors for ignorance are negative. For example:

(...) Ignorance is blindness; to know is to see. Or knowledge is power; ignorance is helplessness and impotence, lgnorance is no defense (...) (Smithson, ibid)

From either metaphors or theorizations, the underpinning assumption is that ignorance is to be reduced (by gaining knowledge or applying logical systems of rules to quantifying and managing it) or banished altogether. However, according to Smithson (2008), there is a potentially interesting but largely unexplored set of linkages between ignorance (and knowledge), emotional responses, moral assessments, and thereby legitimation. Smithson holds that:

(...) ignorance can be used by the ignoramus as a justification for evading culpability or responsibility. In many cultures, education and other forms of knowledge transmission are moralizing projects; so too are ignorance arrangements such as secrecy, privacy, and the protection of innocence (...).(Smithson, ibid)

\section{The Knowledge-Ignorance Contradiction in Education}

Without ignorance, there would be no knowledge, without knowledge, there would be no ignorance. Common metaphors for ignorance conspicuously show its contradiction with knowledge and present them as the opposites in a struggle. For instance, Ignorance is blindness; to know is to see. Or knowledge is power; ignorance is helplessness and impotence. Here comes the contradiction:

To be ignorant is to be blind (...) to have knowledge is to see. Blindness is the absence of sight, while sight is the 
absence of blindness. In other words, someone whose sense of sight is normal and can see, cannot be blind. Likewise, a blind person has no eyes and therefore he/she cannot be asked to see. This way, blindness and sight are opposites. Likewise, an ignorant person cannot be said to have knowledge of a claim to an entity or circumstances. A knowledgeable person would be expected to show abilities to a claim or circumstances that an ignorant person cannot. This sets ground for the law of contradiction and its application in the conceptualization of education.

From dialectical account, each contradiction and each of its aspects have their respective characteristics; this is the particularity and relativity of contradiction. In given conditions, opposites possess identity, and consequently can coexist in a single entity and can transform themselves into each other; this again is the particularity and relativity of contradiction. But the struggle of opposites is ceaseless, it goes on both when the opposites are coexisting and when they are transforming themselves into each other, and becomes especially conspicuous when they are transforming themselves into one another; this again is the universality and absoluteness of contradiction. In education, as already discussed, this contradiction exists between knowledge and ignorance. The more people realize how ignorant they are, there more they seek knowledge. It happens that the more knowledge a person has, there he/she discovers what is not known and continues to seek more. This becomes the basis of doing research in education. As argued, in research, ignorance precedes knowledge.

Zedong (1937) explains the interdependence of the contradictory aspects present in all things and the struggles between these aspects determine the life of all things and push their development forward. There is nothing that does not contain contradiction; without contradiction nothing would exist. Contradiction is the basis of the simple forms of motion (for instance, mechanical motion) and still more so of the complex forms of motion. This paper argues that with regard to education, the struggle between knowledge and ignorance justifies the very practice of education. If human beings were born with all knowledge, or if all things were known, there will be ignorance, the basis upon which there will be knowledge. The very search of knowledge is an attempt to eliminate ignorance. This implies that ignorance sets stage for knowledge seeking. Should ignorance cease to be, hence no struggle with knowledge, then education as a transmission of knowledge will equally cease to be. This way, the existence of education, hence its life depends on the struggle and contradiction between knowledge and ignorance.

\section{Idea of Educated Versus Ignorant Person}

The contradiction between knowledge and ignorance manifests itself in the idea of educated and ignorant persons, which sets a stage for a struggle. To be ignorant must distinguished from being uneducated. An ignorant person is not necessarily uneducated. Equally, a knowledgeable person may not necessarily be educated. The contradiction in education with regard to knowledge and ignorance arises on the fact that education transmits knowledge. Taking lessons from dialectical claims, knowledge cannot be without ignorance and neither can ignorance be without knowledge. This having been said, it follows that an educated person has acquired knowledge thereby moving towards elimination of ignorance. This way, a contradiction is found in the qualities of the two people, that is, the knowledgeable and the ignorant.

Mohanan (ibid) argues that an educated person should have the capability to enhance and modify his/her knowledge and thinking abilities on an ongoing basis so as to cope with novel situations and to cope with them in a more successful manner. Michigan State University (www.msu.edu/unit/provost/Educated_Person.htm) posts that an educated person is someone who has learned how to acquire, analyze, synthesize, evaluate, understand, and communicate knowledge and information. Balogun (2008) holds that an educated person is one who shows evidence of a well integrated personality, meaning being economically prudent, socially and politically competent, morally acceptable and intellectually and culturally sophisticated. Being economically prudent means being economically efficient in the sense of possessing skills and knowledge that earns a means of survival as well as making a contribution to the common good. This reflected on dialectical lines would imply that a person without knowledge would fall short of the idea of an educated person.

A person cannot be said to be educated yet he/she has no knowledge. This is so because education seeks a transmission of knowledge. An ignorant person has no knowledge of a proposition or a claim under consideration. To know in dialectical terms implies not to know. To transmit knowledge implies to transmit ignorance as not transmit knowledge leads to its non-acquisition. Someone who has not acquired knowledge is squarely ignorant, since he/she lacks knowledge. Therefore, in the process of acquiring knowledge, people also acquire ignorance. Here is an example:

$A$ has knowledge of $Y$, but has no knowledge of $Z$. $B$ has no knowledge of $Y$ and $Z$. In this illustration, $B$ is in a state of tabula rasa; this is to say that $B$ has a blank mind with regard to knowledge of $Y$ and $Z$. Now $A$ transmits his 
knowledge to $B$. Since $A$ has knowledge of $Y$, to some degree, $B$ is expected to acquire knowledge of $Y$. But $A$ has no knowledge of $Z$, hence ignorant of $Z$. It follows that $B$ will not have knowledge of $Z$ as this has not been transmitted to $B$. In this relationship, $A$ has transmitted to $B$, the knowledge of $Y$, and at the same time, the ignorance of $Z$. The lack of knowledge of $Z$ in both $A$ and $B$, does not mean that $Z$ does not exist, but only that $A$ and $B$ are ignorant of it. The process of transmission of knowledge between $A$ and $B$, has been equally a transmission of ignorance between $A$ and $B$. It is on this basis that this paper finds the practice of education as transmission of knowledge and ignorance.

From the above discussion and illustration, this paper contents that a definition of education that excludes ignorance is biased. Looking at how people have applied ignorance in different situations of life, ignorance cannot be a villain, but an asset. This conceptualization contradicts the call to eliminate ignorance. This paper has shown that ignorance can be used by the ignoramus as a justification for evading culpability or responsibility. In many cultures, education and other forms of knowledge transmission are moralizing projects; so too are ignorance arrangements such as secrecy, privacy, and the protection of innocence. Numerous social relations depend on systematic ignorance arrangements. Trust and politeness are obvious examples. The cohesion and smooth operation of many organizations and institutions hinge on ignorance arrangements, and not only (or even typically) for maintaining power differentials.

The very concept of research, for example, presupposes conscious ignorance about the object of research at the outset; otherwise there is nothing to research (Smithson, ibid)

Specialization is a social ignorance arrangement. The stereotypical explanation for specialization is that it arises when there is too much for any one person to learn everything. But viewed from an adaptational standpoint, specialization is an example of spreading risk in three respects. First, the risks of direct learning (versus vicarious learning, which is less risky) are spread across the population by diversifying learning. Second, the risk of being ignorant about crucial matters is spread by diversifying ignorance. Third, the risks associated with bearing knowledge also are diversified. As with any kind of risk spreading, specialization requires various forms of social cooperation to yield these benefits.

Privacy is an example of another kind of social ignorance arrangement. Privacy often has been construed as control over access by others to information, mainly about the self. Privacy involves a consensual and essentially cooperative ignorance arrangement, whereas secrecy is unilaterally imposed. Trust "entails a state of perceived vulnerability or risk." A primary source of that risk is a requirement that the truster remain partially ignorant about the trustee. Trust is not about concealing information from others, but trust relationships (for example, friendships) do entail a kind of privacy. If a person believes another is monitoring them or insisting that they self-disclose or account for their actions, that person will infer that the other does not trust them.

Trust, therefore, is both an example of a social relation that requires tolerance of ignorance and also trades undesired uncertainty (the risk of being exploited) against desired uncertainty (freedom to seize opportunities for new relations) (Smithson, ibid).

It is evident from the above observations that ignorance is part and parcel of people's lives. It exists even as hidden knowledge. Like in the case of privacy and secrecy, one is simply hiding some knowledge from others. If the call to eliminate ignorance is entertained, then by implication there would be no privacy and secrecy, elements that people have claimed as their rights. As a result, ignorance has been subjectively portrayed in metaphors and related expression to the extent that ignorance is taken to be a villain. As shown and argued in this paper, such a conceptualization of ignorance is erroneous and ignorance must be recognized as exisingt in any process that claims to transmit knowledge, or any state/proposition that lays claim to knowledge. As argued elsewhere in this paper, this is the basis of justifying the inclusion of ignorance in the definition of education. A balanced definition of education would then be:

Education is the process of transmitting knowledge, ignorance, skills and attitudes so as to lead to the acquisition of desirable abilities and qualities.

\section{Conclusion}

It is the conclusion of this paper that ignorance is an essential element in people's life as it is the source of every continued inquiry and discovery. Ignorance exists as a contradiction of knowledge, the struggle between the two being the stem of their very existence. Transmission of knowledge cannot exclude ignorance, which signifies the absence of knowledge. Without ignorance, there would be no knowledge, without knowledge, there would be no ignorance. A definition of education that encompasses knowledge necessarily implies ignorance. This way, if such definition excludes ignorance, then it is a biased definition that does not fully provide for the understanding of education.

\section{References}


Akinpelu, J. (1969). An Introduction to Philosophy of Education. London: Macmilan

Aubery, E. (1999). Hupernostic Teaching and the Place of Ethical and Religious Values in Higher Education. Pittsburg. University of Pennsylvania.

Balogun, A. B (2008). The Idea of "an Educated Person" in the Contemporary African Thought. Ogun State, Nigeria .Olabisi Onabanjo University.

Gwanfogbe M.B. (2006) Changing Regimes and the Development of Education inCameroon, Bamenda: Unique Printers.

Harvard University Graduate School of Education (http://www.gse.harvard.edu/news-impact/2012/04/watch-the-askwith-forum-livedefining-the-educated-person/Definingthe Educated Person).

Lakoff, G . Johnson, M. (1980). Metaphors We Live By . Chicago. University of Chicago Press.

Lenin, V. I. (1958), "On the Question of Dialectics", Collected Works, Vol. XXXVIII Russ. ed., Moscow,

Michigan State University (www.msu.edu/unit/provost/Educated_Person.htm

Mohanan.K. P. Who is an Educated Person? http:// www.cdtl.nus.edu.sg/ publications / educated/ intro.htm

Omona, A.M (1998). Sociology of Education. Department of Distance Education, Makerere University Kampala.

Peters, R.S. (1967). The Concept of Education. Oxford. Oxford University Press

Schofield, H. (1972). The Philosophy of Education. Oxford. Oxford University Press.

Smithson, M. J (2008). "Social Theories of Ignorance" in: Proctor, R.N and Londa, S. (ed). The Making and Unmaking of Ignorance. Stanford. Stanford University Press.

The 1828 edition of Noah Webster's An American Dictionary of the English Language http://modernedfailedus.blogspot.com/ 2005/12/times-have-changed.html)

Warren, C., B. Laslett, B. (1977). "Privacy and Secrecy: A Conceptual Comparison," Journal of Social Issues 33.

Zedong M. (1937). On Contradiction. Accessed from: books.google.com/books=0873326822. 